Case Report

\title{
Autoimmune Lymphoproliferative Syndrome and Epstein-Barr Virus-Associated Lymphoma: An Adjunctive Diagnostic Role for Monitoring EBV Viremia?
}

\author{
Romina Pace ${ }^{1}$ and Donald C. Vinh ${ }^{2}$ \\ ${ }^{1}$ Department of Medicine, McGill University Health Centre, Montreal, QC, Canada H3G 1A4 \\ ${ }^{2}$ Division of Infectious Diseases, Division of Allergy \& Clinical Immunology (Department of Medicine), \\ Department of Medical Microbiology, Department of Human Genetics, McGill University Health Centre, Montreal General Hospital, \\ 1650 Cedar Avenue, Rm A5-156, Montreal, QC, Canada H3G 1A4
}

Correspondence should be addressed to Donald C. Vinh; donald.vinh@mcgill.ca

Received 30 May 2013; Accepted 2 July 2013

Academic Editors: N. Martinez-Quiles, A. E. Tebo, M. Trendelenburg, and A. Vojdani

Copyright (C) 2013 R. Pace and D. C. Vinh. This is an open access article distributed under the Creative Commons Attribution License, which permits unrestricted use, distribution, and reproduction in any medium, provided the original work is properly cited.

Background. Autoimmune lymphoproliferative syndrome (ALPS) is a genetic disorder of lymphocyte homeostasis due to defects in FAS-mediated apoptosis. ALPS is characterized by childhood onset of chronic lymphadenopathy and splenomegaly, autoimmunity, an expanded population of double-negative T cells (DNTCs), and an increased risk of lymphoma. This propensity for lymphoma in ALPS is not well understood. It is possible that lymphomagenesis in some of these patients may result from Epstein-Barr virus (EBV) infection exploiting the defective T-cell surveillance resulting from impaired FAS-mediated apoptosis. Case Presentation. We report the first case, to our knowledge, of lymphoma in a patient with ALPS that was clinically heralded by progressively increasing EBV viremia. We discuss its practical implications and the possible immune pathways involved in the increased risk for EBV-associated lymphoproliferative disorders in ALPS patients. Conclusion. In patients with ALPS, distinguishing chronic lymphadenopathy from emerging lymphoma is difficult, with few practical recommendations available. This case illustrates that, at least for some patients, monitoring for progressively increasing EBV viremia may be useful.

\section{Background}

Autoimmune lymphoproliferative syndrome (ALPS) is a Mendelian disorder of lymphocyte homeostasis caused by defects in FAS-mediated apoptosis [1]. The majority of cases to date are due to heterozygous germline mutations in the APT1 (TNFRSF6) gene, which encodes the cell surfaceexpressed transmembrane receptor, FAS (CD95) [2]. Ligation of FAS by its cognate ligand (FAS ligand; FAS-L) triggers Band T-lymphocyte apoptosis. Mutations in FAS, FAS-L, or in the downstream molecules that constitute the apoptosisinducing complex (i.e., Caspase-10) impair programmed cell death of lymphocytes. Consequently, ALPS is characterized by childhood onset of chronic ( $>6$ months) noninfectious, nonmalignant lymphadenopathy and splenomegaly, associated with an increased circulating number of a unique Tcell population that is $\mathrm{CD} 3+$ and expresses the $\alpha / \beta$ receptor but is CD4- and CD8-, the so-called double-negative T cells (DNTCs). DNTCs are considered elevated when they are $>1.5 \%$ of total lymphocytes or $>2.5 \%$ of CD3+ lymphocytes in the setting of normal or elevated lymphocyte counts [2]. Additional diagnostic criteria for ALPS include polyclonal hypergammaglobulinemia, elevated vitamin B12 levels, and elevated immune biomarkers in plasma (e.g., soluble FAS-L, IL 10, and IL-18) [2]. The clinical course of ALPS is marked by increased rates of autoimmunity and of malignancy. In particular, individuals with germline FAS mutations have risks for Hodgkin's lymphoma (HL) and non-HL of 51- and 
14-fold, respectively, above the general population [3]. This predisposition to lymphoma in ALPS is not fully understood. Failure of the FAS apoptotic pathway is apparently necessary, likely by providing an expanded lymphoid pool at risk for clonal transformation. However, the temporal delay between the onset of ALPS manifestations and that of lymphoma suggests a requirement for additional oncogenic events. EpsteinBarr virus (EBV) is a ubiquitous lymphotropic $\gamma$-herpesvirus. With its ability to establish lifelong latency in B cells and its growth transformation capacity, EBV is associated with various lymphoproliferative disorders (LPD). Diagnoses of some EBV-related LPD are facilitated by quantitative monitoring of EBV viral load (VL) in blood. We report the first case, to our knowledge, of lymphoma in a patient with ALPS that was heralded by progressively increasing EBV viremia and discuss its clinical implications.

\section{Case Presentation}

A 33-year-old French-Canadian male was referred for reevaluation of chronic lymphadenopathy and splenomegaly since the age of 4, when he had been diagnosed with "chronic EBV illness" based on serology. He had no history of unexplained fever, liver dysfunction, cytopenia, mosquito bite reactions, or hydroa vacciniforme, features that would suggest the nosologic entity "chronic active EBV." Based on elevated serum vitamin B12 level, polyclonal hypergammaglobulinemia and $15 \%$ DNTC, the diagnosis of ALPS was confirmed by identification of a heterozygous c.784A $>$ T mutation in TNFRSF6, resulting in p.I262F mutation in the intracellular death domain of FAS (GeneDx). Initial whole blood EBV VL was 0 copies/mL. Baseline positron-emission tomography (PETCT) demonstrated diffuse hypermetabolic lymphadenopathy with enlarged but not hypermetabolic spleen. Right axillary lymph node biopsy revealed paracortical expansion with DNTCs and increased number of $\mathrm{EBV}^{+} \mathrm{B}$ cells (by EBVencoded small RNA (EBER)) but no lymphoma. At 1-year followup, he was noted to have asymptomatic EBV viremia (Figure 1). Serial monitoring of the viral load demonstrated an initial decline and then progressive increase in EBV viremia followed by worsening lymphadenopathy, without constitutional symptoms. PET-CT demonstrated nodal foci with significantly increased metabolic activity. Lymph node excision in toto was required because of a nondiagnostic percutaneous biopsy; histopathological analysis and molecular rearrangement studies demonstrated a composite lymphoma (i.e., follicular lymphoma, diffuse large B-cell lymphoma (DLBCL), and classical HL). Chemotherapy with Rituximab, to target the $\mathrm{EBV}^{+} \mathrm{B}$ cells, cyclophosphamide, doxorubicin, vincristine and, prednisone (R-CHOP) has led, to a dramatic improvement in his lymphomas and concomitant EBV viral load.

\section{Discussion}

Propensity for lymphoma in ALPS is not well understood. The role of EBV, if any, is undefined. In the general population, EBV has been implicated as the causative agent in a number of lymphomas, including $\mathrm{HL}$ and certain DLBCL, albeit in only a proportion of cases [4]. Certain primary (e.g., Wiskott-Aldrich syndrome) [5] or secondary immunodeficiencies (e.g. transplant; HIV) are known to be at increased risk for EBV-LPD; again, only an unexplained select subgroup of patients is affected. In the original ALPSassociated lymphoma cohort at the National Institutes of Health, 10 of 130 subjects heterozygous for germline FAS mutation developed lymphoma: three (two of B cell origin, one of $\mathrm{T}$ cell) were EBV-positive on pathology by in situ hybridization (Table 1) [3]. Although the clinical penetrance of ALPS in subjects with germline FAS mutation is variable, lymphoma appeared to principally occur in only those who manifest some or all features of ALPS, an observation echoed by Neven et al. [6]. More recently, 20 subjects constituted the ALPS/lymphoma NIH cohort [2], of which 4 had EBVassociated disease [7], while Neven et al. [6] reported one EBV-positive case among their cohort of 7 subjects. Thus, $\sim 15-30 \%$ of lymphomas in patients with ALPS appear to be EBV-related.

Surveillance for lymphoma in ALPS patients is challenging for several reasons: the waxing/waning course of lymphadenopathy; the difficulty in distinguishing nonmalignant lymphadenopathy from malignancy on metabolic imaging [9]; and limitations associated with repeat biopsies of multiple lymph nodes (e.g., feasibility; sampling error) during a potentially insidious course of illness that may span decades. While B-type symptoms (i.e., fever, night sweats, weight loss, and itching) may be suggestive, it may not always be present (as in this case) or may manifest only later in the lymphomagenesis process. Thus, there is a need for complementary tumor-specific biomarkers. Given that some lymphoma cases in ALPS patients appear to be EBV associated, monitoring EBV VL in blood may be a useful adjunctive tool to guide the need for more aggressive diagnostic investigations. Routine monitoring of EBV VL in blood is well established as a predictor and assessment of treatment response of PTLD, as well as other EBV-related malignancies [10]. In our case, initial lymph node biopsy demonstrated cells latently infected with EBV by EBER in situ hybridization but without evidence of lymphoma; there was no concomitant detectable EBV viremia. EBV VL was then monitored because of the historical diagnosis of "chronic EBV illness," serendipitously illustrating a progressively increasing EBV VL in blood metachronous to lymphomatous transformation. Although no causality is implied, this case suggests that monitoring EBV VL in at least some ALPS patients may be a useful proxy test for certain types of lymphoproliferative malignancies. Nomura et al. recently reported the detection of EBV viremia in 3 patients initially diagnosed with CAEBV but subsequently found to be heterozygous for FAS mutations, suggesting that they, in fact, had ALPS [8]. However, in these patients, only a single EBV VL was reported and none had malignancy (although lymph node histopathology was not reported). Thus, if EBV VL proves clinically useful in diagnosing or predicting lymphoma in some patients with ALPS, it may be through dynamic increases on serial measurements, rather than from single values, as is seen in PTLD. 


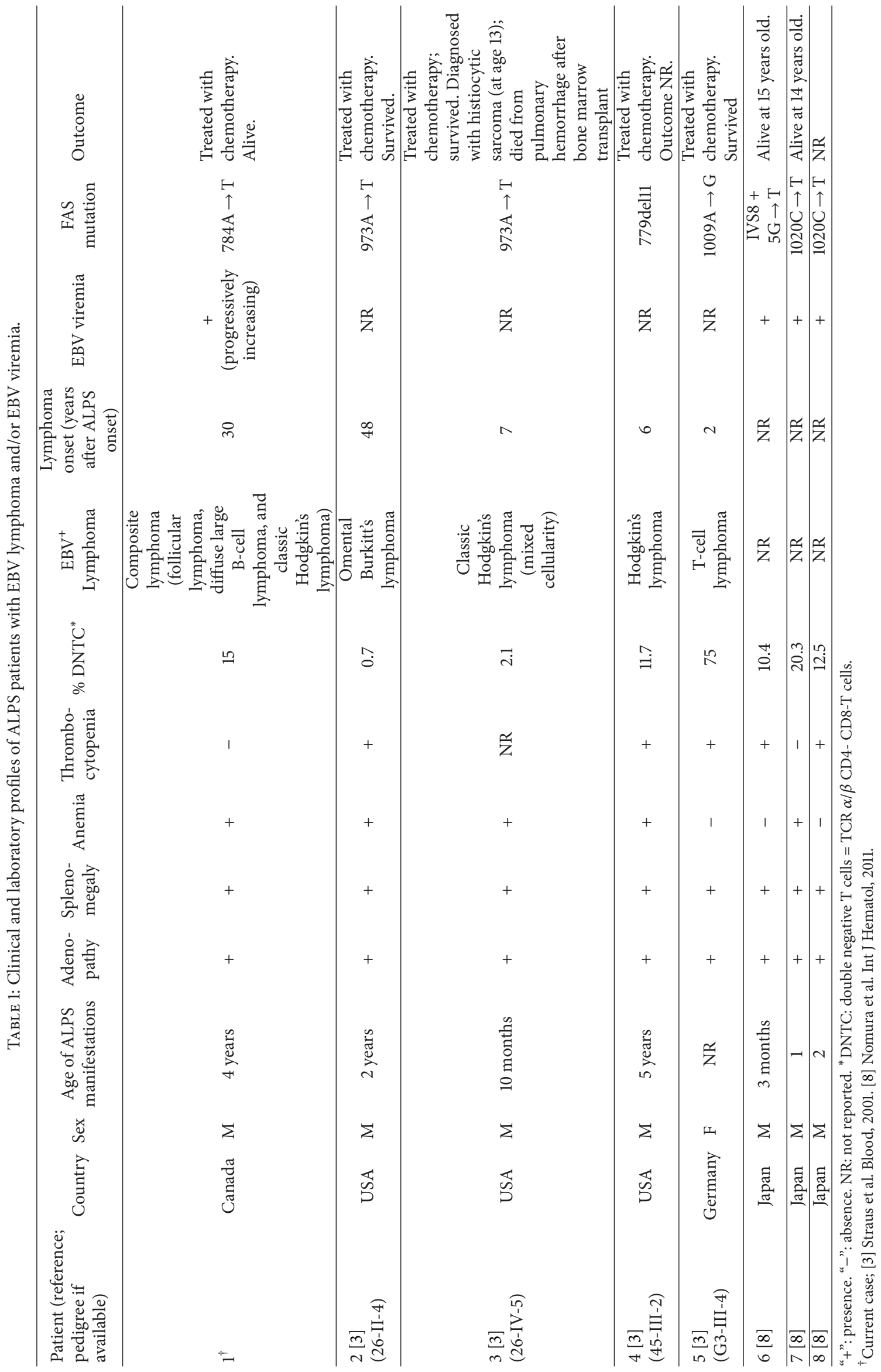




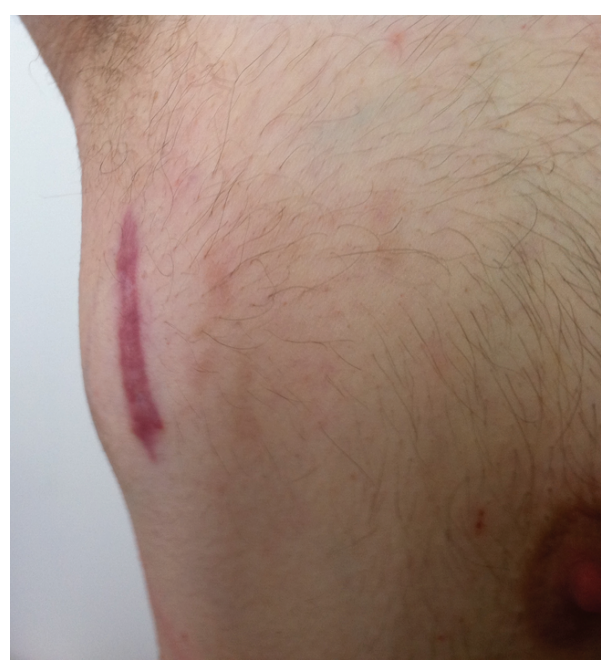

(a)

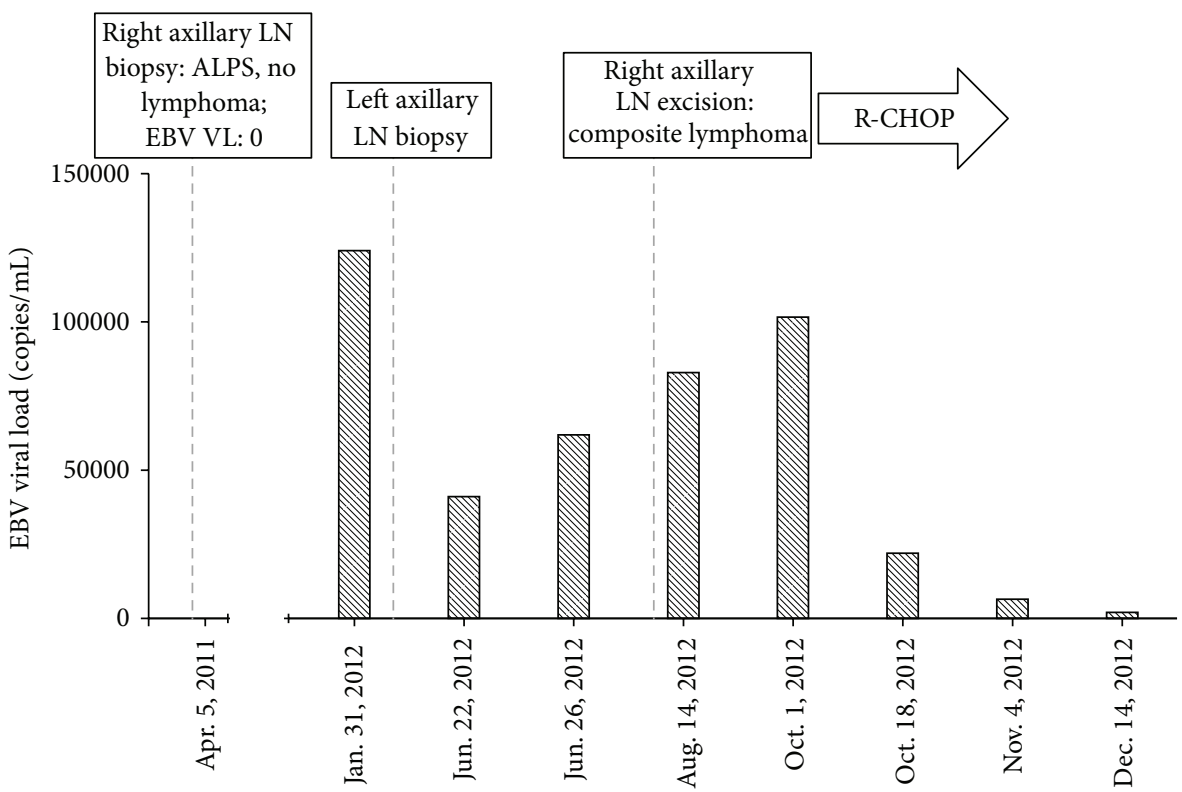

(c)

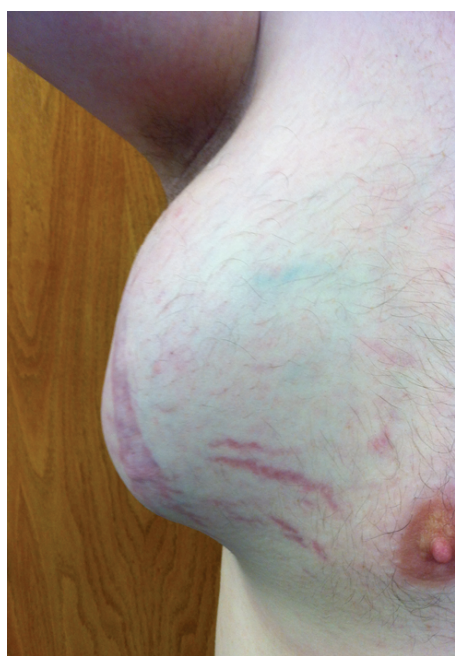

(b)

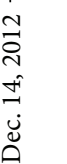

FIGURE 1: EBV viremia and EBV ${ }^{+}$lymphoma in ALPS. A 33-year-old male with ALPS-Ia developed asymptomatic worsening lymphadenopathy (Right axillary lymph node in July 2011 (a) and June 2012 (b)), with progressively increasing EBV viral load (VL). He was found to have a composite $\mathrm{EBV}^{+}$lymphoma. (c) Monitoring of whole blood EBV VL demonstrates an increasing viremia with concomitant identification of lymphoma and improvement in viremia with (rituximab, cyclophosphamide, doxorubicin, vincristine, and prednisone; RCHOP) chemotherapy.

\section{Conclusion}

This case and the literature review suggest that patients with ALPS may have increased susceptibility to EBV-associated lymphomas. Further, this case fortuitously illustrates that at least in some patients with ALPS, serial monitoring of EBV VL may be a useful noninvasive biomarker to monitor for lymphomatous transformation. Further studies are needed to confirm these findings.

\section{List of Abbreviations}

ALPS: Autoimmune Lymphoproliferative Syndrome DNTCs: Double-negative T cells

EBV: $\quad$ Epstein-Barr virus

LPD: Lymphoproliferative disorder

HL: Hodgkin's lymphoma

VL: Viral load

PET-CT: Baseline positron emission tomography 
EBER: EBV-encoded small RNA

DLBCL: Diffuse large B-cell lymphoma

R-CHOP: Rituximab, cyclophosphamide, doxorubicin, vincristine, and prednisone

PTLD: Posttransplant lymphoproliferative disorder

CAEBV: Chronic active Epstein-Barr virus.

\section{Conflict of Interests}

The authors do not have a direct financial relation with any commercial identity mentioned in this paper that might lead to a conflict of interests for any of the authors. D. C. Vinh has received an unrestricted educational grant and speaking fees from CSL Behring Canada.

\section{Authors' Contribution}

D. C. Vinh was involved in the medical care of this patient. D. C. Vinh and R. Pace drafted the paper. All authors read and approved the final paper.

\section{Acknowledgment}

The authors are grateful for the patient and his family for participation in this study.

\section{References}

[1] W. Al-Herz, A. Bousfiha, J. L. Casanova et al. et al., "Primary immunodeficiency diseases: an update on the classification from the international union of immunological societies expert committee for primary immunodeficiency," Frontiers in Immunology, vol. 2, p. 54, 2011.

[2] V. K. Rao and J. B. Oliveira, "How I treat autoimmune lymphoproliferative syndrome," Blood, vol. 118, no. 22, pp. 5741-5751, 2011.

[3] S. E. Straus, E. S. Jaffe, J. M. Puck et al., "The development of lymphomas in families with autoimmune lymphoproliferative syndrome with germline Fas mutations and defective lymphocyte apoptosis," Blood, vol. 98, no. 1, pp. 194-200, 2001.

[4] M. K. Gandhi, E. Lambley, J. Burrows et al., "Plasma EpsteinBarr virus (EBV) DNA is a biomarker for EBV-positive Hodgkin's lymphoma," Clinical Cancer Research, vol. 12, no. 2, pp. 460-464, 2006.

[5] R. Amnueilaph, P. Boongird, M. Leechawengwongs, and A. Vejjajiva, "Heroin neuropathy," The Lancet, vol. 1, no. 7818, pp. 1517$1518,1973$.

[6] B. Neven, A. Magerus-Chatinet, B. Florkin et al., "Asurvey of 90 patients with autoimmune lymphoproliferative syndrome related to TNFRSF6 mutation," Blood, vol. 118, no. 18, pp. 47984807, 2011.

[7] G. Venkataraman, K. L. McClain, S. Pittaluga, V. K. Rao, and E. S. Jaffe, "Development of disseminated histiocytic sarcoma in a patient with autoimmune lymphoproliferative syndrome and associated rosai-dorfman disease," American Journal of Surgical Pathology, vol. 34, no. 4, pp. 589-594, 2010.

[8] K. Nomura, H. Kanegane, K. Otsubo et al., "Autoimmune lymphoproliferative syndrome mimicking chronic active EpsteinBarr virus infection," International Journal of Hematology, vol. 93, no. 6, pp. 760-764, 2011.
[9] V. K. Rao, J. A. Carrasquillo, J. K. Dale et al., "Fluorodeoxyglucose positron emission tomography (FDG-PET) for monitoring lymphadenopathy in the autoimmune lymphoproliferative syndrome (ALPS)," American Journal of Hematology, vol. 81, no. 2, pp. 81-85, 2006.

[10] H. Kimura, Y. Ito, R. Suzuki, and Y. Nishiyama, "Measuring Epstein-Barr virus (EBV) load: the significance and application for each EBV-associated disease," Reviews in Medical Virology, vol. 18, no. 5, pp. 305-319, 2008. 


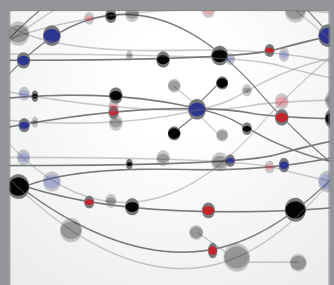

The Scientific World Journal
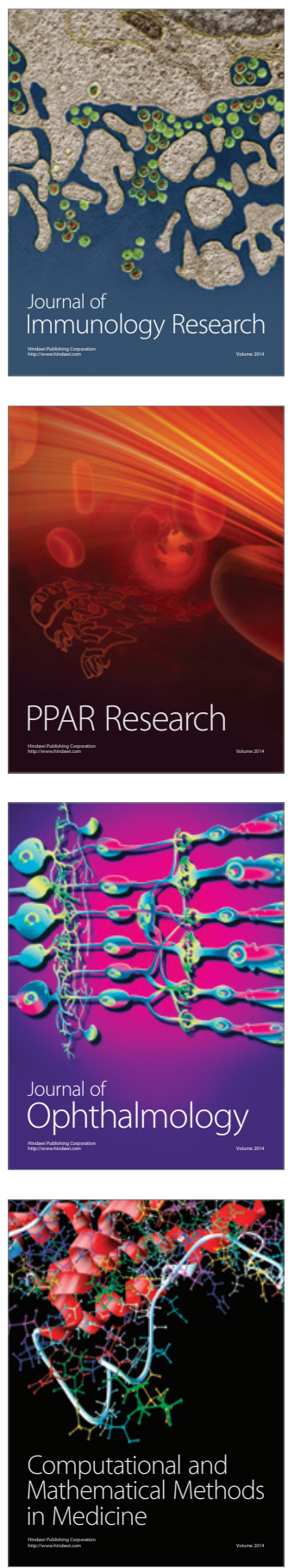

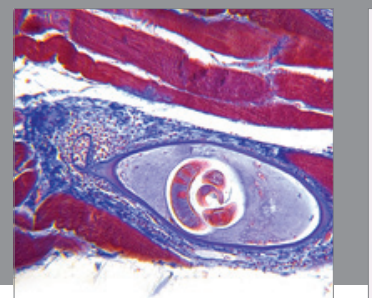

Gastroenterology

Research and Practice
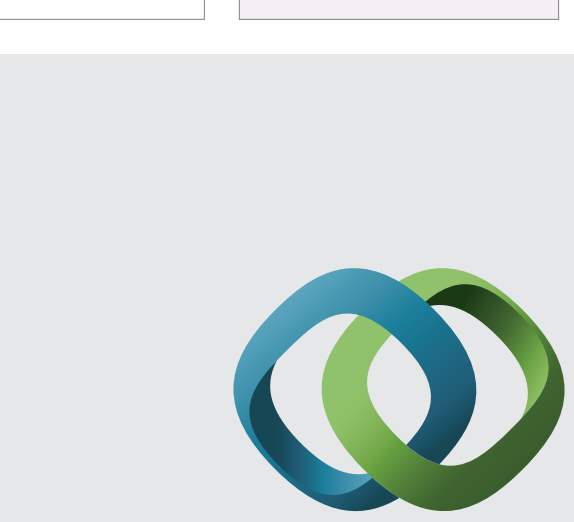

\section{Hindawi}

Submit your manuscripts at

http://www.hindawi.com
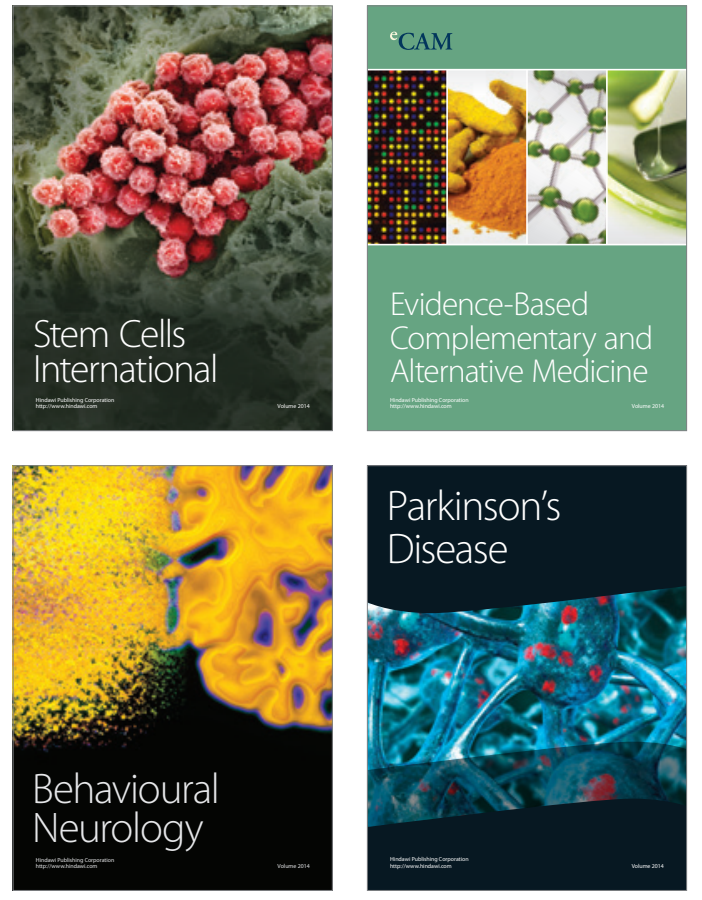
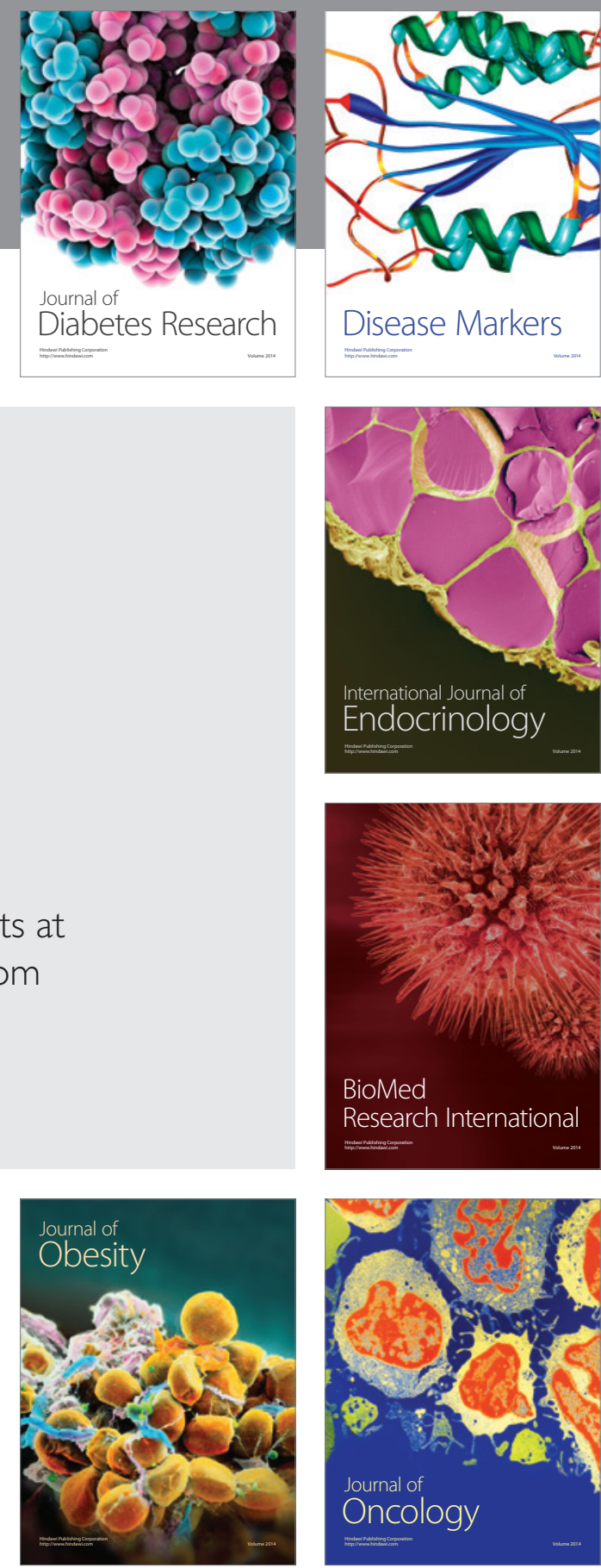

Disease Markers
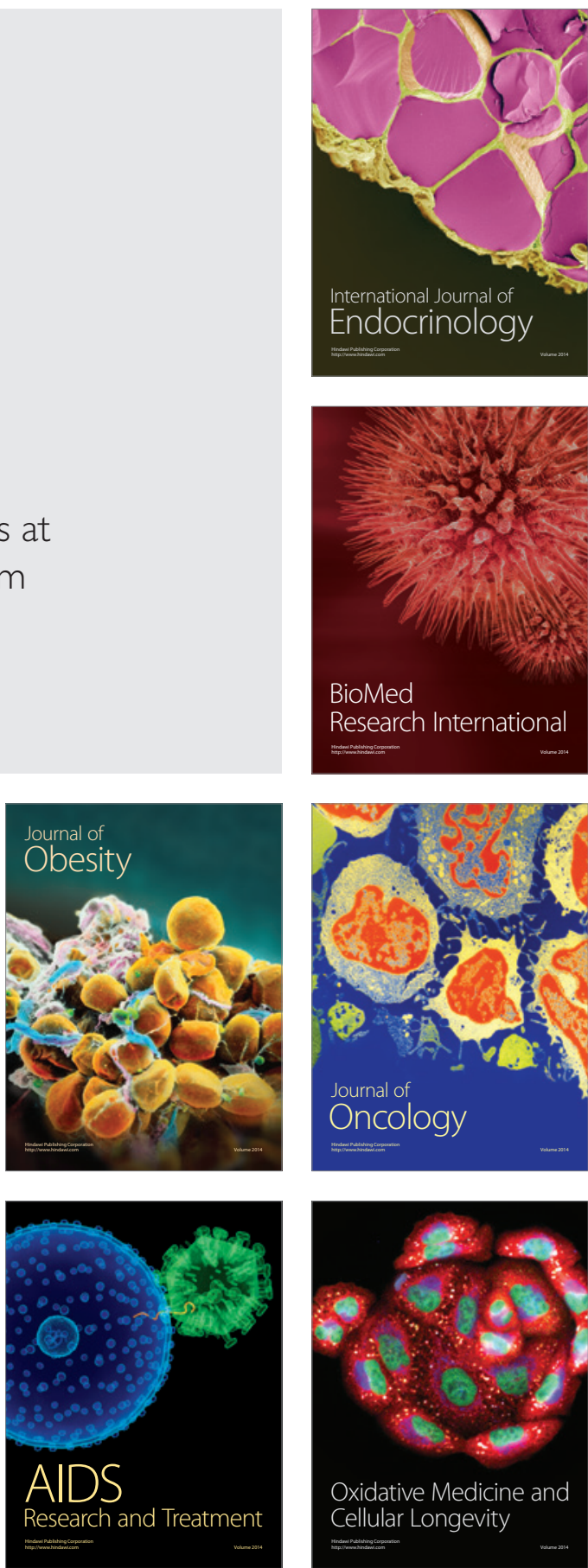